\title{
Evolution in Action: David Sloan Wilson's Forthcoming The Neighborhood Project
}

\author{
Adam M. Goldstein
}

Published online: 20 January 2011

(C) Springer Science+Business Media, LLC 2011

The Neighborhood Project: Using Evolution to Improve my City, One Block at a Time (Wilson 2011), David Sloan Wilson's next book, is set for publication in July 2011, by Little Brown. Wilson has been hard at work on Evolutionary Studies, known also as "EvoS," which is a general approach to teaching and research that extends evolutionary thinking to all aspects of human life. The Neighborhood Project complements Evolution for Everyone (Wilson 2007), which was published at roughly the same time that SUNY Binghamton's EvoS was in the early stages of implementation under Wilson's directorship. While Evolution for Everyone represents Wilson's thought at the point at which his experiences with EvoS were beginning, The Neighborhood Project represents them after the point at which the program's early successes now afford him the time to reflect. The difference between the two books is exemplified by their subtitles. Compare "How Darwin's Theory Can Change the Way We Think About Our Lives," to "Using Evolution to Improve my City"on the one hand, merely thinking about how evolution

\footnotetext{
A. M. Goldstein $(\varangle)$

Department of Philosophy, Iona College,

715 North Avenue,

New Rochelle, 10801 NY, USA

e-mail: z_californianus@shiftingbalance.org
}

can change our lives, and on the other, changing them.

The prose style of The Neighborhood Project is striking. In Evolution for Everyone, Wilson often takes a first-person perspective, but almost as often, he places himself in the background. The Neighborhood Project is deeply personal, narrated in a strong first-person voice. "For me, science is a medium for listening and reflecting upon the human condition, much like religion and literature.... Science can expand our capacities for listening and reflecting. Indeed, unless we use these expanded capacities, there is no hope of solving the problems of modern existence." He adds: "I'm studying [my city] Binghamton, not as an aloof scientist, but because I think my expertise can make a difference." Along the way, Wilson explains, among other things, economic theories, religion, literature, psychology, and of course, evolutionary science, but these are framed by Wilson's personal story.

Wilson's shift in emphasis and perspective toward the first person and the practical does not conceal even the smallest measure of retreat from long-held views about the compatibility of science and religion, the central importance of social life and group affinity in biological evolution, or the usefulness of evolutionary psychology. He remains committed to a theme of his past work: although there are evolutionary explanations for religion, literature and other arts, and altruistic feelings or behavior, such explanations do not explain them away. Those who differ with Wilson about these issues will nonetheless benefit from reading The Neighborhood Project, not only because the narrative is so engaging but also because Wilson explains his ideas in a more powerful and nuanced manner as a result of his EvoS work. Those who have found Wilson's views 
appealing in the past will benefit from The Neighborhood Project for the same reasons. Finally, those not familiar with Wilson's previous work should start reading Evolution for Everyone immediately so that they will be able to appreciate the excitement and be well prepared to join the many animated discussions that The Neighborhood Project will no doubt provoke!

\section{References}

Wilson DS. Evolution for everyone: how Darwin's theory can change the way we think about our lives. New York: Delacorte; 2007.

Wilson DS. The neighborhood project: using evolution to improve my city, one block at a time. New York: Little, Brown; 2011 (in press). 